\title{
Clinical significance of the De Ritis ratio for detecting prostate cancer in a repeat prostate biopsy
}

\author{
Heon $\mathrm{Ha}^{1}{ }^{1}$, Jae-Wook Chung ${ }^{2}$ (1), Yun-Sok Ha ${ }^{1,3}$, Seock Hwan Choi ${ }^{1,3}$ (D), Jun Nyung Lee ${ }^{2,3}$ (D), Bum Soo Kim ${ }^{1,3}$, \\ Hyun Tae Kim ${ }^{2,3}$ (D), Tae-Hwan Kim ${ }^{2,3}$ (D), Ghil Suk Yoon ${ }^{3,4}$, Tae Gyun Kwon ${ }^{2,3}$, Sung Kwang Chung ${ }^{1,3}$, \\ Eun Sang Yoo ${ }^{1,3}$ \\ 'Department of Urology, Kyungpook National University Hospital, Daegu, ${ }^{2}$ Department of Urology, Kyungpook National University Chilgok Hospital, Daegu, \\ ${ }^{3}$ Department of Urology, School of Medicine, Kyungpook National University, Daegu, ${ }^{4}$ Department of Pathology, Kyungpook National University Chilgok Hospital, \\ Daegu, Korea
}

Purpose: We evaluated factors predicting a positive repeat biopsy result in patients with an initial negative prostate biopsy result. Materials and Methods: This study included 124 patients in whom prostate cancer (PCa) was not detected in the initial transrectal ultrasound-guided prostate biopsy and who underwent repeat biopsy from January 2011 to December 2017. Patients without PCa in both initial and repeat prostate biopsies were designated as group $1(n=82)$, and those in whom PCa was detected on a repeat prostate biopsy were designated as group $2(n=42)$. Among group 2 patients, 6 had insignificant PCa according to the Epstein criteria and were combined with group 1 patients to make up group $A(n=88)$. Patients with significant PCa were categorized as group $B(n=36)$. We compared clinicopathologic characteristics between the groups.

Results: Multivariate analysis showed that age $(p=0.018)$ and detection of atypical small acinar proliferation (ASAP) or $\geq 3$ cores of high-grade prostatic intraepithelial neoplasia (HGPIN) $(p=0.011)$ on the initial biopsy were predictive factors for a positive result on a repeat biopsy. When we compared group $A$ and group $B$, age $(p=0.004)$ and the De Ritis ratio $(p=0.024)$ were significantly higher in group $B$ in the multivariate analysis.

Conclusions: Age and the detection of ASAP or $\geq 3$ cores of HGPIN on the initial biopsy were associated with detection of PCa on a repeat biopsy. Age and the De Ritis ratio were found to be predictive factors for the detection of clinically significant PCa on a repeat biopsy.

Keywords: Biopsy; Prostatic neoplasms; Transaminases

This is an Open Access article distributed under the terms of the Creative Commons Attribution Non-Commercial License (http://creativecommons.org/licenses/by-nc/4.0) which permits unrestricted non-commercial use, distribution, and reproduction in any medium, provided the original work is properly cited.

\section{INTRODUCTION}

Prostate cancer $(\mathrm{PCa})$ is one of the most common cancers in males in the United States and the third most common cause of cancer-related death [1]. The incidence of PCa is relatively lower in Korea than in Western countries. However, the age-adjusted incidence rate of $\mathrm{PCa}$ in Korea increased from 10.1 per 100,000 people in 2002 to 27.0 per 100,000 people

Received: 19 April, 2019 • Accepted: 30 June, 2019

Corresponding Author: Eun Sang Yoo (iD https://orcid.org/0000-0002-7442-6886

Department of Urology, Kyungpook National University Hospital, School of Medicine, Kyungpook National University, 130 Dongdeok-ro, Jung-gu, Daegu 41944 , Korea

TEL: +82-53-420-5843, FAX: +82-53-421-9618, E-mail: uroyoo@knu.ac.kr 
in 2012 [2]. Prostate-specific antigen (PSA) is the most novel serum marker used for the early detection and management of PCa. The diagnosis of PCa is precipitated by a persistent increase in the serum PSA level, which triggers the performance of a prostate biopsy.

However, PSA testing is controversial owing to its relative lack of cancer specificity. In a previous study, approximately $20 \%$ to $30 \%$ of patients with potential PCa were not identified as having the disease at the time of the initial prostate biopsy [3]. The results of a prostate biopsy may include PCa, benign prostatic hyperplasia, high-grade prostatic intraepithelial neoplasia (HGPIN), and atypical small acinar proliferation (ASAP) [4]. HGPIN and ASAP have been recognized as premalignant lesions and are potential risk factors for PCa [5]. HGPIN is characterized by a distinctive architectural arrangement of cellular proliferations within preexisting ducts and glands as in $\mathrm{PCa}$, but without the complete disruption of the basal cell layer and stromal invasion that are observed in carcinoma [6]. ASAP is commonly considered atypical foci suspicious for but not diagnostic of malignancy [7]. It has been reported that the cancer detection rate on repeat biopsy in patients with HGPIN ranges from $25 \%$ to $79 \%$. The cancer detection rate on repeat biopsy in patients with ASAP ranges from $21 \%$ to $60 \%$ [8]. However, there is no consensus about the management of patients with HGPIN and ASAP.

Thus, after the initial biopsy, considerable numbers of patients are confronted with repeat biopsy. Although prostate biopsy is generally considered a safe and effective procedure, the possibility of (severe) complications and the cost associated with repeat biopsy should not be ignored. Patients may also experience fear and discomfort with repeat biopsy. Therefore, careful selection of patients for repeat biopsy is necessary to increase the probability of a positive biopsy and to reduce the number of unnecessary and uncomfortable biopsies.

Attention to the De Ritis ratio has recently increased in the urologic field. Aminotransaminases, including aspartate aminotransferase (AST) and alanine aminotransferase (ALT), are well-known biomarkers of hepatic function. ALT is a hepatocyte-specific enzyme expressed in the liver at high concentrations, whereas AST is expressed not only in the liver but also in various other tissues such as the skeletal muscle, kidney, heart, and brain [9-11]. Several previous studies have reported the efficacy of aminotransaminases as a prognostic factor for various cancers. Focusing on these characteristics of the De Ritis ratio, we evaluated whether the De Ritis ratio may be a meaningful factor for predicting $\mathrm{PCa}$.
Proper interpretation of follow-up PSA testing is necessary after an initial negative biopsy result, and management of patients with suspected PCa, such as those with abnormal results on a digital rectal examination, increasing PSA, or premalignant findings at the initial biopsy, is also important. Although many studies have been conducted of the factors that may predict $\mathrm{PCa}$ in repeat biopsy, the results of these studies are controversial. Furthermore, selection of subjects for repeat biopsy among patients who are not subject to active surveillance is clinically important in the treatment of $\mathrm{PCa}$. In this study, we evaluated factors predictive of a positive repeat biopsy result in patients with an initial negative prostate biopsy result.

\section{MATERIALS AND METHODS}

\section{Study population}

Data from 211 patients in whom PCa was not detected in the initial transrectal ultrasound (TRUS)-guided prostate biopsy and who subsequently underwent repeat biopsy at our center between January 2011 and December 2017 were included and retrospectively reviewed. The exclusion criteria were as follows: 1) lack of blood test results including AST and ALT levels, 2) previously diagnosed hepatic disease (e.g, hepatitis, hepatic cellular cancer, liver cirrhosis), and 3) lack of regular follow-up. Finally, 124 patients were enrolled in this study. Initial prostate biopsy was performed when the serum PSA level was $>3.0 \mathrm{ng} / \mathrm{mL}$, when there were abnormal findings on the digital rectal examination, or both. Additional repeat biopsies were done when the result of the first biopsy included ASAP or $\geq 3$ cores of HGPIN [12,13] or when the serum PSA level was continuously increased. This study was approved by the Ethics Committee of the Kyungpook National University School of Medicine (approval number: KNUH 2018-11-009). Written informed consent of this study was disapplied due to its retrospective characteristics.

\section{Prostate biopsy protocol}

In our center, more than 800 prostate biopsies were performed in a year. All prostate biopsies were performed by using TRUS under local anesthesia. Most patients underwent TRUS-guided prostate biopsy in the outpatient department without admission. For the initial biopsy, 12-core prostate biopsy was performed, and for repeat biopsy, >18core prostate biopsy was performed.

\section{Study design}

This study was a retrospective single-center trial. Fig. 1 


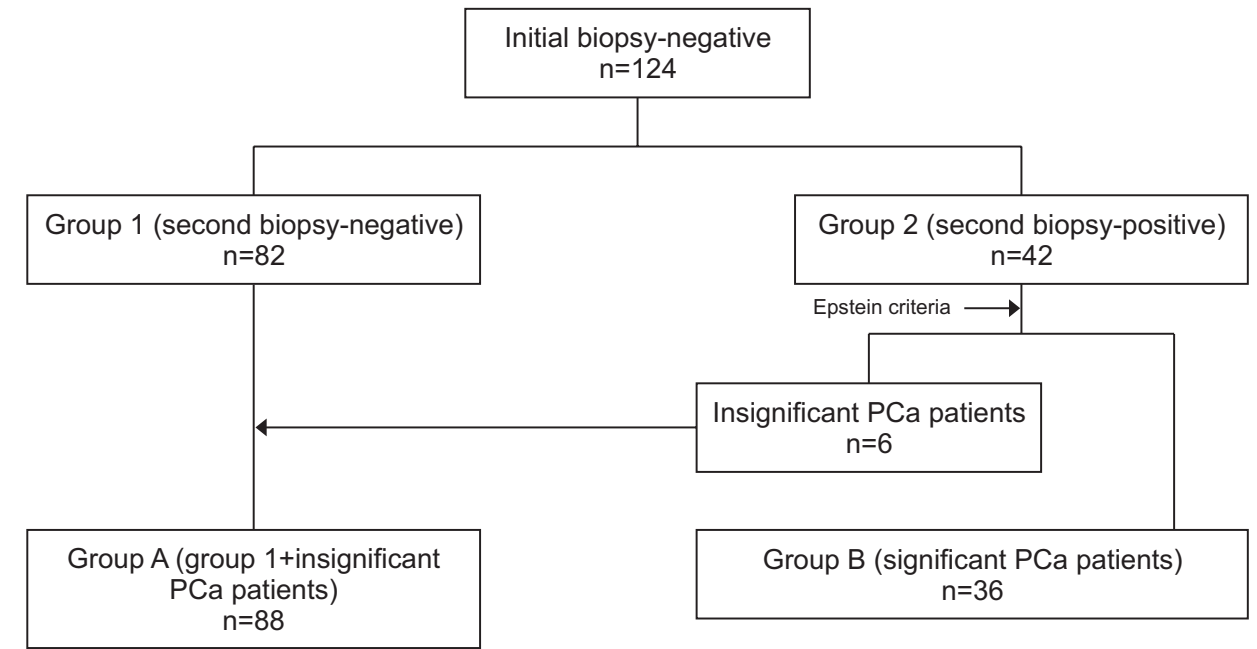

Fig. 1. Study algorithm. Group 1, without prostate cancer ( $\mathrm{PCa}$ ) detection in initial and repeat prostate biopsy; group 2 , PCa detected in repeat prostate biopsy; group $A$, benign or low-risk prostate cancer; group $B$, non-low-risk prostate cancer. shows the study algorithm. We grouped the patients into group 1 ( $\mathrm{n}=82)$, which included those without $\mathrm{PCa}$ in both the initial and repeat prostate biopsy, and group $2(n=42)$, which included those in whom PCa was detected in the repeat prostate biopsy. Among group 2 patients, 6 had insignificant PCa according to the Epstein criteria [14,15] (Stage T1c, PSA density $[\mathrm{PSAD}] \leq 0.15 \mathrm{ng} / \mathrm{mL}$ per gram, Gleason score $\leq 6$, fewer than three positive cores, and $<50 \%$ of cancer involvement in any core). We further subclassified the patients into group A and group B. Group A ( $\mathrm{n}=88)$ was defined as group 1 plus the 6 patients with insignificant PCa. Group B $(n=36)$ comprised the remaining patients with significant $\mathrm{PCa}$.

Clinicopathologic characteristics including age, initial PSA level, PSA level on repeat prostate biopsy, prostate volume, and the time interval between initial and repeat prostate biopsies were compared among the groups. Prostate volume was calculated by using TRUS. PSAD was defined as the PSA level divided by the prostate volume on initial biopsy. PSA velocity (PSAV) was calculated by dividing the time between the initial and the repeat biopsies with the change in the PSA level. The ratio of the serum activity of AST to that of ALT was defined as the De Ritis ratio [16]. The De Ritis ratio was calculated at least 4 weeks before the initial prostate biopsy.

\section{Statistical analysis}

The continuous variables of groups A and B were compared by using Student's t-test. Comparisons of noncontinuous variables were performed by using the chi-square test. The predictive accuracy of the De Ritis ratio was assessed by using receiver operating characteristic curve analysis. The cutoff value was set as the value that maximizes the sum of sensitivity and specificity. Comparisons between group A and group B were done in the same way as comparisons between groups 1 and 2. Multiple logistic regression models were used for multivariate analysis. Statistical analysis was performed by using SPSS 16.0 for Windows (SPSS Inc., Chicago, IL, USA), and p-values $<0.05$ were considered statistically significant.

\section{RESULTS}

A total of 124 patients were enrolled. The clinicopathologic characteristics of groups $1(n=82)$ and $2(n=42)$ are shown in Table 1. The mean patient age was $64.19 \pm 7.83$ years. The mean interval from the first to the repeat biopsy was $21.44 \pm 16.14$ months. The mean serum PSA level at the time of the initial biopsy was $6.78 \pm 3.30 \mathrm{ng} / \mathrm{mL}$ and that at the repeat biopsy was $8.83 \pm 8.91 \mathrm{ng} / \mathrm{mL}$, respectively. The mean prostate volume was $50.29 \pm 19.59 \mathrm{~cm}^{3}$. The mean PSAV was $1.51 \pm 10.29 \mathrm{ng} \cdot \mathrm{mL}^{-1} \cdot$ year $^{-1}$, and the mean PSAD was $0.14 \pm 0.08$ $\mathrm{ng} / \mathrm{mL}^{2}$. Patients in group 2 were significantly older than those in group 1 ( $66.36 \pm 6.18$ vs. $63.07 \pm 8.38$ years, $\mathrm{p}=0.013)$. However, the biopsy interval, serum PSA level at the initial and repeat biopsies, prostate volume, PSAD, and PSAV were not significantly different between the groups. Of the patients, $21.0 \%$ (26/124) showed ASAP or $\geq 3$ cores of HGPIN on the initial biopsy. The detection rate of ASAP or $\geq 3$ cores of HGPIN on the initial biopsy was higher in group 2 than in group 1 (35.7\% [15/42] vs. 13.4\% [11/82], p=0.011). The mean De Ritis ratio was $1.42 \pm 0.05$. The $D e$ Ritis ratio was significantly higher in group 2 than in group 1 ( $1.55 \pm 0.31$ vs. $1.35 \pm 0.56$, $\mathrm{p}=0.011$ ). Multivariate analysis showed that age (odds ratio [OR], 1.082; 95\% confidence interval [CI] 1.017-1.152; $\mathrm{p}=0.018$ ), and detection of ASAP or $\geq 3$ cores of HGPIN (OR, 3.707; 95\% CI, 1.354-10.147; $p=0.011$ ) on the initial biopsy were the only predictive factors for a positive repeat biopsy.

The clinicopathologic characteristics of group A $(n=88)$ 
Table 1. Comparison of clinicopathologic characteristics of group 1 and group 2

\begin{tabular}{|c|c|c|c|c|c|c|}
\hline \multirow{2}{*}{ Characteristic } & \multirow{2}{*}{ Total } & \multirow{2}{*}{ Group 1} & \multirow{2}{*}{ Group 2} & \multicolumn{3}{|c|}{ p-value } \\
\hline & & & & Univariate & Multivariate & OR $(95 \% \mathrm{Cl})$ \\
\hline Total number of patients & $124(100.0)$ & $82(66.1)$ & $42(33.9)$ & & & \\
\hline Age (y) & $64.19 \pm 7.83$ & $63.07 \pm 8.38$ & $66.36 \pm 6.18$ & 0.013 & 0.018 & $1.082(1.017-1.152)$ \\
\hline Interval (mo) & $21.44 \pm 16.14$ & $21.59 \pm 16.62$ & $21.17 \pm 15.36$ & 0.892 & & \\
\hline PSA of initial biopsy (ng/mL) & $6.78 \pm 3.30$ & $6.74 \pm 3.02$ & $6.87 \pm 3.83$ & 0.825 & & \\
\hline PSA of repeat biopsy (ng/mL) & $8.83 \pm 8.91$ & $8.90 \pm 10.48$ & $8.68 \pm 4.57$ & 0.895 & & \\
\hline Prostate volume $\left(\mathrm{cm}^{3}\right)^{\mathrm{a}}$ & $50.29 \pm 19.59$ & $51.30 \pm 18.34$ & $48.34 \pm 21.94$ & 0.430 & & \\
\hline PSA velocity $\left(\mathrm{ng} \cdot \mathrm{mL}^{-1} \cdot\right.$ year $\left.^{-1}\right)$ & $1.51 \pm 10.29$ & $1.74 \pm 11.90$ & $1.08 \pm 6.15$ & 0.738 & & \\
\hline PSA density (ng/mL $\left.{ }^{2}\right)$ & $0.14 \pm 0.08$ & $0.14 \pm 0.08$ & $0.15 \pm 0.08$ & 0.230 & & \\
\hline Pathology of initial biopsy & & & & 0.011 & & \\
\hline Benign & $66(53.2)$ & $46(56.1)$ & $20(47.6)$ & & & \\
\hline $1 \leq \mathrm{HGPIN} \leq 2$ & $32(25.8)$ & $25(30.5)$ & $7(16.7)$ & & 0.478 & $0.687(0.244-1.936)$ \\
\hline ASAP or HGPIN $\geq 3$ & $26(21.0)$ & $11(13.4)$ & $15(35.7)$ & & 0.011 & 3.707 (1.354-10.147) \\
\hline De Ritis ratio $^{b}$ & $1.42 \pm 0.05$ & $1.35 \pm 0.56$ & $1.55 \pm 0.31$ & 0.011 & 0.100 & $1.937(0.881-4.258)$ \\
\hline
\end{tabular}

Values are presented as number (\%) or mean \pm standard deviation.

Group 1, without prostate cancer (PCa) detection in initial and repeat prostate biopsy; group 2, PCa detected in repeat prostate biopsy; OR, odds ratio; $\mathrm{Cl}$, confidence interval; PSA, prostate-specific antigen; HGPIN, high-grade prostatic intraepithelial neoplasia; ASAP, atypical small acinar proliferation.

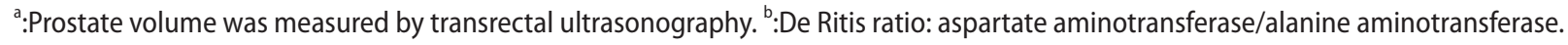

Table 2. Comparison of clinicopathologic characteristics of group A with group B

\begin{tabular}{|c|c|c|c|c|c|c|}
\hline \multirow{2}{*}{ Characteristic } & \multirow{2}{*}{ Total } & \multirow{2}{*}{ Group A } & \multirow{2}{*}{ Group B } & \multicolumn{3}{|c|}{ p-value } \\
\hline & & & & Univariate & Multivariate & OR $(95 \% \mathrm{Cl})$ \\
\hline Total number of patients & $124(100.0)$ & $88(71.0)$ & $36(29.0)$ & & & \\
\hline Age (y) & $64.19 \pm 7.83$ & $63.13 \pm 8.19$ & $66.75 \pm 6.27$ & 0.010 & 0.004 & $1.107(1.032-1.187)$ \\
\hline Interval (mo) & $21.44 \pm 16.14$ & $21.18 \pm 16.24$ & $22.08 \pm 16.12$ & 0.779 & & \\
\hline PSA of initial biopsy (ng/mL) & $6.78 \pm 3.30$ & $6.82 \pm 3.14$ & $6.69 \pm 3.71$ & 0.843 & & \\
\hline PSA of repeat biopsy $(\mathrm{ng} / \mathrm{mL})$ & $8.83 \pm 8.91$ & $8.85 \pm 10.13$ & $8.78 \pm 4.87$ & 0.968 & & \\
\hline Prostate volume $\left(\mathrm{cm}^{3}\right)^{\mathrm{a}}$ & $50.29 \pm 19.59$ & $52.26 \pm 18.16$ & $45.48 \pm 22.28$ & 0.081 & & \\
\hline PSA velocity $\left(\mathrm{ng} \cdot \mathrm{mL}^{-1} \cdot\right.$ year $\left.{ }^{-1}\right)$ & $1.52 \pm 10.29$ & $1.38 \pm 11.90$ & $1.85 \pm 4.51$ & 0.820 & & \\
\hline PSA density (ng/mL $\left.{ }^{2}\right)$ & $0.14 \pm 0.08$ & $0.13 \pm 0.08$ & $0.16 \pm 0.09$ & 0.062 & & \\
\hline Pathology of initial biopsy & & & & 0.066 & & \\
\hline Benign & $66(53.2)$ & $48(54.5)$ & $18(50.0)$ & & & \\
\hline $1 \leq \mathrm{HGPIN} \leq 2$ & $32(25.8)$ & $26(29.5)$ & $6(16.7)$ & & & \\
\hline ASAP or HGPIN $\geq 3$ & $26(21.0)$ & $14(15.9)$ & $12(33.3)$ & & & \\
\hline De Ritis ratio ${ }^{b}$ & $1.42 \pm 0.50$ & $1.34 \pm 0.56$ & $1.61 \pm 0.24$ & 0.001 & 0.024 & $2.670(1.135-6.280)$ \\
\hline
\end{tabular}

Values are presented as number (\%) or mean \pm standard deviation.

Group A, benign or low-risk prostate cancer; group B, non-low-risk prostate cancer; OR, odds ratio; Cl, confidence interval; PSA, prostate-specific antigen; HGPIN, high-grade prostatic intraepithelial neoplasia; ASAP, atypical small acinar proliferation.

${ }^{a}$ :Prostate volume was measured by transrectal ultrasonography. ${ }^{b}$ :De Ritis ratio: aspartate aminotransferase/alanine aminotransferase.

and group $\mathrm{B}(\mathrm{n}=36)$ are shown in Table 2 . When we classified the patients into those with benign or insignificant $\mathrm{PCa}$ (group A) and those with significant PCa (group B), patients in group B were significantly older than those in group A (66.75 \pm 6.27 vs. $63.13 \pm 8.19$ years, respectively, $p=0.010$ ). The biopsy interval, serum PSA level at the initial and repeat biopsies, prostate volume, PSAV, and the pathologic findings on the initial biopsy were not significantly different between groups A and B. The De Ritis ratio was significantly higher in group B than in group A ( $1.61 \pm 0.24$ vs. $1.34 \pm 0.56$, $\mathrm{p}=0.001)$. Multivariate analysis showed that age (OR, 1.107; 95\% CI, 1.032-1.187; $\mathrm{p}=0.004)$ and the De Ritis ratio (OR, 2.670; 95\% CI, 1.135-6.280; $\mathrm{p}=0.024$ ) were significantly higher in group B.

\section{DISCUSSION}

A repeat biopsy should be performed when a patient 
with a negative result in the initial biopsy continues to be suspected of having PCa, owing to an increasing PSA level, abnormal findings on a digital rectal examination, or a suspicious lesion on magnetic resonance imaging. Many patients with a negative result in the initial biopsy are often identified as having PCa in a repeat biopsy, and it is important to identify these patients. Furthermore, it is also important to select patients from those who are not undergoing active surveillance as candidates for repeat biopsy.

Previous studies have shown that the probability of PCa is high in patients with ASAP or HGPIN in the initial biopsy. There is considerable debate about whether HGPIN is truly a precancerous lesion with the potential to progress to PCa. However, the current evidence is also in conflict with the variable recommendations on the timing of repeat prostate biopsy. Epstein and Herawi [12], in their review article, concluded that patients with HGPIN do not have an increased risk for PCa compared with those with benign disease, and they recommended that repeat biopsy is not required within the first year of diagnosis of HGPIN. In contrast, Merrimen et al. [17] suggested performing repeat biopsy at 1 year for patients with multifocal disease and active surveillance thereafter. Dorin et al. [18] reported that one-third of patients with ASAP who were diagnosed as having $\mathrm{PCa}$ on a repeat biopsy within 1 year underwent radical prostatectomy and one-quarter underwent radiotherapy, suggesting that early repeat biopsy does identify clinically significant disease. In contrast, Warlick et al. [19] reported that only $17.3 \%$ of patients had Gleason 7 or higher disease at a median follow-up of $>4$ years after an initial diagnosis of ASAP. Leone et al. [20] reported that 17\% had an intermediate risk of cancer and $8 \%$ had high-grade $\mathrm{PCa}$ on repeat biopsy and recommended active surveillance with monitoring of PSA and digital rectal examination changes for patients with ASAP. In this study, 42 patients (33.9\%) were diagnosed as having $\mathrm{PCa}$ in the repeat biopsy. Of these 42 patients, 6 patients (14.3\%) were determined to have insignificant PCa according to the Epstein criteria. These 6 patients are potential candidates for active surveillance.

Recent studies have reported on the efficacy of the De Ritis ratio for determining urogenital malignancy. Lee et al. [21] reported that an increased AST/ALT ratio is significantly associated with worse postoperative survival in patients surgically treated for localized clear cell renal cell carcinoma. Gorgel et al. [10] reported that an increased De Ritis ratio is an independent prognostic factor in patients with bladder cancer who undergo radical cystectomy. Moreover, Cho et al. [22] reported that an increased De Ritis ratio is a prognostic factor for shorter cancer-specific survival and overall survival in patients with upper-tract urothelial carcinoma after radical nephroureterectomy. For PCa, the only trial that evaluated the significance of the De Ritis ratio in localized PCa was introduced in 2010, in which an elevated De Ritis ratio seemed to be related to higher Gleason score $\mathrm{PCa}$ [11]. The growth of well-differentiated PCa is thought to be dependent on testosterone. On the contrary, resistance to androgens is associated with poorly differentiated and rapidly proliferating PCa. Poorly differentiated PCa would behave aggressively, with a larger percentage of high-grade tumors, compared with well-differentiated cancer and therefore have a higher recurrence rate after RP. Schatzl et al. [23] found that higher Gleason sum PCa was associated with decreased circulating testosterone levels. Hepatic dysfunction is associated with decreased peripheral circulating testosterone secondary to its conversion to estrogens. Theoretically, greater conversion to estrogens would then favor the growth of poorly differentiated $\mathrm{PCa}$, while providing inadequate stimulation for the proliferation of well-differentiated PCa. Recently, Wang et al. [24] reported that a higher De Ritis ratio could predict worse pathologic outcomes and higher biochemical recurrence in patients with localized PCa. However, there have been few studies on the predictors of PCa diagnosis in prostate biopsy. In our study, the De Ritis ratio (OR, 2.670; 95\% CI, 1.135-6.280; $\mathrm{p}=0.024$ ) was significantly higher in group $\mathrm{B}$ in the multivariate analysis.

However, it remains unclear why the De Ritis ratio is high in various cancers. A possible explanation may be that AST is expressed in a greater variety of tissues compared with ALT, and AST levels may be higher in cancer cells that exhibit higher proliferative status and tissue damage than in normal tissues. Another hypothesis is related to the glucose metabolism in cancer cells. Warburg [25] found that cancer cells produce energy primarily through glycolysis, as opposed to normal cells that use oxidative phosphorylation for energy production. Glycolysis is quicker and readily provides energy in the form of ATP required by rapidly proliferating cancer cells [26]. DeBerardinis et al. [27] suggested that in cancer cells, AST plays an important role in aerobic glycolysis by allowing the nicotinamide adenine dinucleotide hydride that had been generated in the cytoplasm to be effectively relocated within the mitochondria. It has also been reported that AST may be a valid molecular target for the development of anti-neoplastic agents [28] Thornburg et al. [28] observed definite antimetabolic effects due to inhibition of AST, as well as suppressed proliferation of breast cancer cells with AST inhibition. Even with these academic insufficiencies, our study is the first to predict clinically significant $\mathrm{PCa}$ in a repeat prostate biopsy by using the De Ritis ratio. 
Our study had some limitations, including its retrospective and single-center design. Moreover, because a considerable number of patients who did not undergo blood tests were excluded, a selection bias did exist. Concerning liver enzymes, in 2008 [29], Lazo et al. [29] studied the short-term variability in results of liver function tests in 1,864 men and women aged 18 years or older who underwent two liver function tests within a short interval. They concluded that more than $30 \%$ of adults with elevated AST, ALT, or bilirubin levels recovered to within the normal range with only resting and that clinicians should be aware of the high intra-individual variability in common liver tests. Furthermore, substantial diurnal variation in aminotransferase can exist [30]. Serum ALT levels can change over time, with the highest activity in the afternoon and the lowest at night and early morning. Therefore, variation in the time of blood testing may affect the variability in aminotransferase level. We did not control the timing or other factors that can influence the results of liver function tests. Also, we did not analyze the second liver function test before the repeat prostate biopsy. Individual alcohol and herb use were not recorded, which is also limitation of our trial. Prospective and multicenter trials should be done in the future.

\section{CONCLUSIONS}

In this study, age and the detection of ASAP or $\geq 3$ cores of HGPIN on the initial biopsy were associated with the detection of PCa on a repeat biopsy. In addition, age and the De Ritis ratio were predictive factors for the detection of clinically significant $\mathrm{PCa}$ on a repeat biopsy. These factors should be considered when planning repeat biopsy.

\section{CONFLICTS OF INTEREST}

The authors have nothing to disclose.

\section{ACKNOWLEDGMENTS}

This research was supported by the Basic Science Research Program through the National Research Foundation of Korea (NRF) and was funded by the Korean government (MSIT) (2014M3A9D3034164), (2016R1C1B1011180), (2018R1C1B5040264) (2019R1A2C1004046) and the Ministry of Trade, Industry and Energy (R0005886).

\section{REFERENCES}

1. Siegel RL, Miller KD, Jemal A. Cancer statistics, 2017. CA Can- cer J Clin 2017;67:7-30.

2. Jung KW, Won YJ, Kong HJ, Lee ES; Community of Population-Based Regional Cancer Registries. Cancer statistics in Korea: incidence, mortality, survival, and prevalence in 2015. Cancer Res Treat 2018;50:303-16.

3. Presti JC Jr, O'Dowd GJ, Miller MC, Mattu R, Veltri RW. Extended peripheral zone biopsy schemes increase cancer detection rates and minimize variance in prostate specific antigen and age related cancer rates: results of a community multipractice study. J Urol 2003;169:125-9.

4. Kim TS, Ko KJ, Shin SJ, Ryoo HS, Song W, Sung HH, et al. Multiple cores of high grade prostatic intraepithelial neoplasia and any core of atypia on first biopsy are significant predictor for cancer detection at a repeat biopsy. Korean J Urol 2015;56:796-802.

5. Iczkowski KA, Chen HM, Yang XJ, Beach RA. Prostate cancer diagnosed after initial biopsy with atypical small acinar proliferation suspicious for malignancy is similar to cancer found on initial biopsy. Urology 2002;60:851-4.

6. Shepherd D, Keetch DW, Humphrey PA, Smith DS, Stahl D. Repeat biopsy strategy in men with isolated prostatic intraepithelial neoplasia on prostate needle biopsy. J Urol 1996;156(2 Pt 1):460-2; discussion 462-3.

7. Montironi R, Scattoni V, Mazzucchelli R, Lopez-Beltran A, Bostwick DG, Montorsi F. Atypical foci suspicious but not diagnostic of malignancy in prostate needle biopsies (also referred to as "atypical small acinar proliferation suspicious for but not diagnostic of malignancy"). Eur Urol 2006;50:666-74.

8. Leite KR, Srougi M, Dall'Oglio MF, Sanudo A, Camara-Lopes LH. Histopathological findings in extended prostate biopsy with PSA $<$ or $=4$ ng/mL. Int Braz J Urol 2008;34:283-90; discussion 290-2.

9. Botros M, Sikaris KA. The de ritis ratio: the test of time. Clin Biochem Rev 2013;34:117-30.

10. Gorgel SN, Kose O, Koc EM, Ates E, Akin Y, Yilmaz Y. The prognostic significance of preoperatively assessed AST/ALT (De Ritis) ratio on survival in patients underwent radical cystectomy. Int Urol Nephrol 2017;49:1577-83.

11. Bañez LL, Loftis RM, Freedland SJ, Presti JC Jr, Aronson WJ, Amling CL, et al. The influence of hepatic function on prostate cancer outcomes after radical prostatectomy. Prostate Cancer Prostatic Dis 2010;13:173-7.

12. Epstein JI, Herawi M. Prostate needle biopsies containing prostatic intraepithelial neoplasia or atypical foci suspicious for carcinoma: implications for patient care. J Urol 2006;175(3 Pt 1):820-34.

13. Merrimen JL, Jones G, Walker D, Leung CS, Kapusta LR, Srigley JR. Multifocal high grade prostatic intraepithelial neoplasia is a significant risk factor for prostatic adenocarcinoma. J Urol 
2009;182:485-90; discussion 490.

14. Kryvenko ON, Carter HB, Trock BJ, Epstein JI. Biopsy criteria for determining appropriateness for active surveillance in the modern era. Urology 2014;83:869-74.

15. Epstein JI, Walsh PC, Carmichael M, Brendler CB. Pathologic and clinical findings to predict tumor extent of nonpalpable (stage T1c) prostate cancer. JAMA 1994;271:368-74.

16. De Ritis F, Coltorti M, Giusti G. An enzymic test for the diagnosis of viral hepatitis: the transaminase serum activities. 1957. Clin Chim Acta 2006;369:148-52.

17. Merrimen JL, Evans AJ, Srigley JR. Preneoplasia in the prostate gland with emphasis on high grade prostatic intraepithelial neoplasia. Pathology 2013;45:251-63.

18. Dorin RP, Wiener S, Harris CD, Wagner JR. Prostate atypia: does repeat biopsy detect clinically significant prostate cancer? Prostate 2015;75:673-8.

19. Warlick C, Feia K, Tomasini J, Iwamoto C, Lindgren B, Risk M. Rate of Gleason 7 or higher prostate cancer on repeat biopsy after a diagnosis of atypical small acinar proliferation. Prostate Cancer Prostatic Dis 2015;18:255-9.

20. Leone A, Gershman B, Rotker K, Butler C, Fantasia J, Miller A, et al. Atypical small acinar proliferation (ASAP): is a repeat biopsy necessary ASAP? A multi-institutional review. Prostate Cancer Prostatic Dis 2016;19:68-71.

21. Lee H, Lee SE, Byun SS, Kim HH, Kwak C, Hong SK. De Ritis ratio (aspartate transaminase/alanine transaminase ratio) as a significant prognostic factor after surgical treatment in patients with clear-cell localized renal cell carcinoma: a propensity score-matched study. BJU Int 2017;119:261-7.

22. Cho YH, Hwang JE, Chung HS, Kim MS, Hwang EC, Jung SI, et al. The De Ritis (aspartate transaminase/alanine transaminase) ratio as a predictor of oncological outcomes in patients after surgery for upper urinary tract urothelial carcinoma. Int Urol Nephrol 2017;49:1383-90.

23. Schatzl G, Madersbacher S, Thurridl T, Waldmüller J, Kramer G, Haitel A, et al. High-grade prostate cancer is associated with low serum testosterone levels. Prostate 2001;47:52-8.

24. Wang H, Fang K, Zhang J, Jiang Y, Wang G, Zhang H, et al. The significance of De Ritis (aspartate transaminase/alanine transaminase) ratio in predicting pathological outcomes and prognosis in localized prostate cancer patients. Int Urol Nephrol 2017;49:1391-8.

25. Warburg O. On the origin of cancer cells. Science 1956;123: 309-14.

26. Elf SE, Chen J. Targeting glucose metabolism in patients with cancer. Cancer 2014;120:774-80.

27. DeBerardinis RJ, Mancuso A, Daikhin E, Nissim I, Yudkoff M, Wehrli S, et al. Beyond aerobic glycolysis: transformed cells can engage in glutamine metabolism that exceeds the requirement for protein and nucleotide synthesis. Proc Natl Acad Sci U S A 2007;104:19345-50.

28. Thornburg JM, Nelson KK, Clem BF, Lane AN, Arumugam $\mathrm{S}$, Simmons A, et al. Targeting aspartate aminotransferase in breast cancer. Breast Cancer Res 2008;10:R84.

29. Lazo M, Selvin E, Clark JM. Brief communication: clinical implications of short-term variability in liver function test results. Ann Intern Med 2008;148:348-52.

30. Kim WR. Clinical implications of short-term variability in liver function test results. Gastroenterology 2008;135:1010-1; discussion 1011-2. 